\title{
Una mirada reflexiva sobre el proceso de formación docente como ejercicio constitutivo de la formación inicial del profesorado
}

\author{
A reflexive approach on teachers' training process as an exercise of constitution of pre- \\ service teacher education
}

Kohen, Micaela; Rodríguez, Edith M.; Pujalte, Alejandro P. y Meinardi, Elsa

GDB - Grupo de Didáctica de la Biología, Centro de Formación e Investigación en Enseñanza de las Ciencias (CEFIEC), Facultad de Ciencias Exactas y Naturales, Universidad de Buenos Aires. Ciudad Autónoma de Buenos Aires, Argentina. emeinardi@fibertel.comar

\section{Resumen}

Los procesos metarreflexivos cobran una dimensión destacada en el marco de la formación inicial del profesorado en la medida en que consolidan el rol docente. En el último tramo de su formación inicial como profesoras de biología, las dos primeras autoras de este trabajo intentan indagar en qué sentido la reflexión sobre su práctica es constitutiva de su propio proceso formativo. Asimismo, analizan de qué manera su participación simultánea en un programa de tutorías -que apunta a brindar apoyo escolar a adolescentes en riesgo social- promueve un conocimiento relacional y contextual que permite repensar las teorías vistas en el profesorado, dotando de sentido a las preguntas acerca de qué ciencia enseñar y para qué. Es así que revisan su propia práctica en las aulas a partir de los marcos teóricos de referencia, estableciendo al mismo tiempo un diálogo con las reflexiones de otros actores involucrados en el mismo programa.

\section{Palabras clave}

Formación docente, jóvenes vulnerables, programa de tutorías, procesos metarreflexivos.

\section{Abstract}

Considered in the context of pre-service teacher education, meta-reflexive processes acquire importance inasmuch as they consolidate the role of teachers. During the last part of their initial education as biology teachers, the first two authors of this artide seek to explore in which sense reflection on their practices results constitutive of their own formation. In the same way, they analyse how their simultaneous participation in a tutoring programme-meant to support students under social risk- promotes a relational and contextual knowledge that allows rethinking the theories considered during their pre-service education as teachers, giving meaning to the questions of which science should be taught and what for. Thus, they interrogate their own practice at school from the theoretical frameworks of reference, establishing at the same time a dialogue with the reflections produced by other actors involved in the same programme.

\section{Keywords}

Pre-service teacher education, students under social risk, tutoring programme, meta-reflexive processes. 


\section{Introducción}

Objetivos

En el presente trabajo relatamos una experiencia que llevamos adelante en la formación inicial del profesorado en la Universidad de Buenos Aires, en Argentina, que persigue la realización de un ejercicio metarreflexivo sobre el propio proceso de formación de los alumnos y alumnas, futuros profesores de enseñanza media y superior, apuntando a dotarlos del conocimiento del contexto de las escuelas que atienden jóvenes en situación de vulnerabilidad social extrema, de manera de mejorar los aprendizajes y los índices de retención escolar.

\section{Marco teórico}

Como señalan numerosos autores, consideramos indispensable una formación inicial y permanente del profesorado que apunte a preparar docentes reflexivos y críticos, capaces de generar buenas prácticas para atender los problemas detectados en las escuelas, especialmente aquellas con jóvenes en situación de vulnerabilidad social. Consideramos impresaindible que todo el proceso de formación se vincule con una reflexión crítica, y que contemple el contexto sociocultural de acción y el mundo emocional del que enseña (Meinardi, 2007). En ese mismo sentido es necesario que el profesorado lleve a cabo una reflexión de tipo metacognitivo que le permita reconocer, evaluar y decidir sobre la necesidad de reelaborar sus concepciones y su práctica. De ahí que los conceptos de cambio profesional y metacognición sean totalmente interdependientes (Copello y Sanmartí, 2001).

Por otro lado, acordamos con la idea de Gomes Lima (2002) acerca de que es importante que el proceso de reflexión ocurra en grupo, para que se establezca la relación de diálogo (...) con todos los involucrados en el proceso educativo; el profesor obtiene el respaldo para trabajar su realidad mediante el diálogo que le posibilitará disponer de huellas que le ayudarán en su práctica pedagógica.

En este proceso, creemos que es especialmente relevante indagar auáles son las concepciones que sobre las finalidades de la enseñanza científica se sustentan en estos ámbitos y para estos jóvenes. Pensamos que las finalidades de la educación cientíica debenían resignificarse en los contextos educativos más desfavorecidos. Se podría afimar que las concepciones acerca de las finalidades educativas atraviesan las prácticas, de forma consciente o no, y que de todas maneras estarían condicionando el hacer pedagógico. Como afirma Perrenoud (2001): "No podemos disociar tan fácilmente las finalidades del sistema educativo de las competencias que se requieren de los docentes. No se privilegia la misma figura del profesor según se desee una escuela que desarrolle la autonomía o el conformismo, (...) el espínitu de indagación o el dogmatismo, el sentido de la cooperación o la competencia, la solidaridad o el individualismo."

De todo lo anterior se desprende que la formación profesional docente debería organizarse alrededor de una práctica que promueva en los educadores y educadoras el desarrollo de un sentido metarreflexivo que les permita abordar las problemáticas de las poblaciones escolares en riesgo. Entendemos que no existe la dicotomía entre teoría y práctica: permanentemente ambos planos se enriquecen y retroalimentan. Por esto consideramos que espacios que posibiliten este "ida y vuelta" deben ser parte de la formación permanente de los profesores. En palabras de Perrenoud, "hay que combatir esta dicotomía y afirmar que la formación es una, en todo momento práctico y teórico a la vez, también reflexivo, crítico y con identidad".

\section{Desarrollo}

El trabajo que presentamos forma parte de la tesis final de formación del profesorado de dos de las autoras, que participan además de un programa de tutorías denominado "Aprender enseñando", 
financiado por el Ministerio de Educación de la Nación, tendiente a prevenir el fracaso y la deserción escolar, en escuelas que atienden jóvenes en situación de vulnerabilidad social extrema (Meinardi, 2009). Todo el proyecto es planificado y seguido por un orientador didáctico, y demanda un trabajo anual que se lleva a cabo en el transcurso de las Didácticas Específicas de la Biología, del profesorado en ciencias en la Universidad de Buenos Aires, Argentina.

Como uno de los objetivos prioritarios de las tareas que realizan durante este trayecto de formación, se establece que los estudiantes del profesorado lleven adelante diversas y múltiples formas de aproximación a los contextos escolares desfavorecidos, donde se vuelve fundamental la indusión educativa de calidad. Se intenta, como parte de esta formación inicial, que los alumnos/as, futuros profesores y profesoras, reflexionen acerca de algunos aspectos vinculados con las instituciones que apuntan a una indusión educativa de los jóvenes más desfavorecidos, porque esto les permitirá comprender la lógica de su funcionamiento, facilitando posteriormente el trabajo profesional en ellas.

En el caso espećfico de las tutorías, los futuros y futuras docentes atienden las dificultades que se les presentan a los alumnos y alumnas durante la resolución de problemas o trabajos prácticos planteados por el o la docente permanente a cargo del curso. Además, el desempeño de la tarea de tutor/a implica asistir al grupo de estudiantes atendiendo los diferentes conflictos que surgen tanto en el aula como en los espacios entre dases. De esta forma el tutor está presente acompañando los procesos educativos sin tener la responsabilidad de "dar la dase", lo que permite mirar lo que sucede desde una óptica diferente a la del docente, muchas veces más atenta a lo contextual.

En este marco, las alumnas-futuras profesoras y, al mismo tiempo tutoras del programa, realizan un trabajo de tesis en el cual se investiga acerca de cómo la doble intervención en la escuela, ya sea mediante el trabajo de residencia pedagógica de formación docente inicial y como tutoras de los jóvenes estudiantes, sirve para modificar sus concepciones respecto del valor social de la educación cient́fica, constituyendo una parte muy significativa de su desarrollo profesional docente. Sumado a esto, en el curso del trabajo se entrevista a otros participantes del programa de tutorías y alumnos/as del profesorado para recabar información acerca de cómo influye en ellos esta experiencia.

\section{Conclusiones}

La brecha social entre los/las docentes que dan dases en estas escuelas y el de sus alumnos y alumnas suele ser muy notoria. En general, las y los docentes en formación se han educado en escuelas de características muy diferentes. Las experiencias escolares que atravesaron distan mucho de la realidad de las instituciones a las que asisten alumnos/as en situación de vulnerabilidad social, como las que atiende el programa de tutorías; por tanto, acercarse a los códigos y costumbres de los/las estudiantes que por ellas transitan debería ser una prioridad si se pretende trabajar de manera eficaz atendiendo a sus necesidades e intereses.

En el trabajo que presentamos se muestra una experiencia de acercamiento de la formación docente inicial a los contextos escolares más desfavorecidos. Al mismo tiempo, la reflexión sobre dicha experiencia sirve para la realización de un ejercicio metacognitivo respecto de cómo el trabajo actúa sobre el propio desarrollo profesional.

Los resultados muestran una toma de posición que se ubica en un lugar de desacuerdo con un tipo de escuela que naturaliza el fracaso de los y las jóvenes que se hallan en situación de vulnerabilidad social extrema. Al mismo tiempo, permiten conduir que es fundamental involucrarse con las instituciones escolares, en la creencia de que se pueden superar ciertas formas cristalizadas 
de trabajo acudiendo a la organización y creatividad de los docentes como agentes productores de cambio.

Sin duda, podemos afirmar que esta experiencia ha contribuido a retroalimentar el proceso de formación docente, en el sentido de habilitar a los futuros profesores y profesoras para trabajar en dichos contextos. Una formación inicial y permanente innovadora, axiológica y creativa prepara a las y los docentes para mirar la realidad con citerios explícitos y para actuar sobre ella. Por un lado, la participación en las tutorías no solo permite generar un vínculo distinto con los estudiantes, sino que también posibilita mirar críticamente las situaciones que ocurren en el aula para poder -en futuras prácticas- tomar decisiones fundadas en estas experiencias. Por otro lado, posibilita pensar en los verdaderos problemas a los que se enfrentan los docentes que trabajan en los contextos más desfavorecidos $y$, al mismo tiempo, repensar los contenidos "aprendidos" en el profesorado a la luz de la realidad escolar.

Consideramos que este ejercicio no sólo es valioso para la formación, sino que además presenta un desafio: promover que los estudiantes puedan realizar el mismo ejercicio metacognitivo sobre su proceso de aprendizaje, contribuyendo a lograr niveles crecientes de autonomía, en función de formar ciudadanos cíticos y reflexivos. Finalmente, este mismo proceso metacognitivo que surge de la formación inicial, atraviesa y dialoga con la formación en servicio, llega al aula a los destinatarios finales (los jóvenes) y se retoma como retroalimentación de los propios procesos reflexivos del equipo de formación docente.

Agradecimientos

A la Agencia Nacional de Investigaciones, Subsidio PICT 26106, y al proyecto UBACyT X 141, por el apoyo a nuestro trabajo.

\section{Bibliografía}

Copello Levy, M. I. y Sanmartí Puig, N. (2001). Fundamentos de un modelo de formación permanente del profesorado de ciencias centrado en la reflexión dialógica sobre las concepciones y las prácticas. Enseñanza de las Ciencias 19 (2), 269-283

Gomes Lima, P. (2002). El educador reflexivo: notas para la orientación de sus prácticas docentes. Educar 30, 57-67

Meinardi, E. (2007). Reflexiones sobre la formación inicial de los profesores de Biología. Revista de Educación en Biología 10 (2), 48-54

Meinardi, E. (2009). Desarrollo profesional docente tendiente a mejorar la retención y la calidad de los aprendizajes en escuelas con jóvenes en situación de vulnerabilidad social extrema. Memoria de Investigación, Universidad de Buenos Aires.

Perrenoud, P. (2001). La formación de los docentes en el siglo XXI. Revista de Tecnología Educativa XIV, no 3, 503-523 